\title{
Une noblesse sous contrôle. Le pouvoir coutumier vu de Rewa (Fidji Est)
}

\section{Émilie Nolet}

\section{(2) OpenEdition \\ 12 Journals}

Édition électronique

URL : http://journals.openedition.org/jso/6552

DOI : 10.4000/jso.6552

ISSN : 1760-7256

\section{Éditeur}

Société des océanistes

\section{Édition imprimée}

Date de publication : 15 décembre 2011

Pagination : 385-402

ISBN : 978-2-85430-037-4

ISSN : 0300-953x

\section{Référence électronique}

Émilie Nolet, «Une noblesse sous contrôle. Le pouvoir coutumier vu de Rewa (Fidji Est) », Journal de la Société des Océanistes [En ligne], 133 | 2e semestre 2011, mis en ligne le 31 décembre 2014, consulté le 20 avril 2019. URL : http://journals.openedition.org/jso/6552 ; DOI : 10.4000/jso.6552

(c) Tous droits réservés 


\title{
Une noblesse sous contrôle. Le pouvoir coutumier vu de Rewa (Fidji Est)
}

par

\author{
Émilie NOLET*
}

\begin{abstract}
RÉSUMÉ
Fidji est un pays mélanésien qui a la particularité d'avoir vu arriver, à partir de 1879, plusieurs vagues de migrants originaires d'Inde. Les relations de la communauté mélanésienne (autochtone) et des Indo-Fidjiens ont été marquées par de fortes tensions, aboutissant à quatre coups d'Etat entre 1987 et 2006. Par ailleurs, Fidji compte au nombre des pays océaniens où un système de chefferies coexiste avec l'Etat mis en place durant la période coloniale (1874-1970). En plus de l'autorité qu'ils ont dans les territoires coutumiers (vanua), les chefs fidjiens ont été étroitement associés aux affaires nationales depuis la cession du pays à la Grande-Bretagne, notamment au travers du grand conseil des chefs, créé par le pouvoir colonial. Depuis une vingtaine d'années, des voix se sont toutefois élevées en faveur d'une plus grande dissociation des deux sphères politiques fidjiennes. En avril 2007, le gouvernement issu du coup d'Etat de décembre 2006 alla jusqu'à interdire les réunions du grand conseil des chefs, en suggérant que les chefs étaient devenus des obstacles à l'édification d'une nation multiethnique et à la stabilité politique. Cette décision fut désapprouvée par une partie des Mélanésiens qui dénonça un comportement irrespectueux vis-à-vis des chefs et parla d'une mise en danger de ses droits et de son identité culturelle. Dans ce contexte, cet article cherche à comprendre comment les débats relatifs au rôle national mais aussi local des chefs peuvent concrètement s'exprimer dans une chefferie fidjienne (vanua), dans la province de Rewa située au sud-est de la grande île de Viti Levu.
\end{abstract}

Mots-ClEFs : Fidji, rôles locaux et nationaux des chefs, construction nationale, statut politique de la « tradition "

Les trente dernières années ont été marquées dans l'ensemble du Pacifique Sud par de profondes transformations, avec un retrait progres-

\begin{abstract}
Fiji is a Melanesian country where several waves of migrants of Indian origin have arrived since 1879. The relationships between the Melanesian (indigenous) and Indo-Fijian communities have been marked by strong tensions leading to four coups d'État between 1987 and 2006. Moreover, in Fiji, like in some other Pacific countries, a traditional political system coexists with the State that was set up during the colonial period (18741970). Besides their authority within customary territories (vanua), Fijian chiefs have been strongly involved with national affairs since the Deed of Cession of Fiji to Great-Britain, notably through the Great Council of Chiefs (which was created by colonial powers). However, for about twenty years, inhabitants have been asking for a greater dissociation of both Fijian political spheres. In April 2007, the government that seized power in a coup d'Etat in 2006 went as far as forbidding the Great Council of Chiefs' meetings. Authorities suggested that chiefs had become obstacles for the building of a multicultural nation and political stability in Fiji. However this decision was disapproved by some Melanesians, who denounced a disrespectful attitude towards their traditional leaders and considered that their cultural identity and rights were endangered. In such context, this article investigates how inhabitants of a Fijian vanua apprehend the role of chiefs at both local and national levels. I study the case of Rewa province, which is localized in the South-Eastern part of the large island of Viti Levu.
\end{abstract}

Keywords: Fiji, local and national roles of chiefs, nation-building, political status of "tradition"

sif des puissances coloniales, une accélération des échanges culturels et économiques et l'émergence de structures politiques et d'une identité

\footnotetext{
* Post-doctorante au Centre de recherche et de documentation sur l'Océanie (CrEDo), Marseille, nolet@pacific-credo.fr
} 
pan-océaniennes. Si les luttes anticoloniales ont pu et peuvent toujours s'appuyer sur des discours valorisant la tradition, le rôle et le contenu des institutions culturelles océaniennes n'en sont pas moins aussi, localement, intensément questionnés : qu’est ce qui, au fond, peut être considéré comme authentique et tout est-il " bon " dans la coutume ? Comment concilier l'affirmation d'une culture nationale et la diversité des expressions culturelles régionales et ethniques ? Est-il possible d'être moderne sans renier ses racines ? Aujourd'hui, on retrouve, tant en Mélanésie qu'en Polynésie et Micronésie, la présence conjointe d'une forme de valorisation et d'instrumentalisation politique de la tradition et d'un discours dépréciatif. Certains Océaniens voyant par exemple dans l'économie des échanges ou dans la présence de systèmes politiques d'origine précoloniale des obstacles majeurs au développement (Fiji Times, 01/04/2009). En parallèle, les processus de globalisation culturelle, politique et économique qui projettent les archipels les plus isolés au cœur du "village mondial " ont entraîné de profondes transformations au sein des organisations sociales et politiques du Pacifique insulaire.

Fidji est un pays mélanésien dont la population inclut deux grands groupes ethniques (la population mélanésienne autochtone et une forte communauté d'origine indienne ${ }^{1}$ ) et où un système de chefferies coexiste avec l'État mis en place à l'époque coloniale (1874-1970). Le système des chefferies, issu d'une réalité antérieure à l'arrivée des colons britanniques, mais dont certains principes ont été créés ou réinventés sous la période coloniale $^{2}$, implique la division du pays en $v a-$ $n u a^{3}$, placés sous l'autorité de chefs détenteurs de titres d'importance variable. D'une façon générale, on distingue à Fidji deux catégories de chefs, ou turaga:

- les hommes et les femmes issus de clans considérés comme nobles, dont le statut est marqué par l'usage d'une particule (Adi pour les femmes, Ratu pour les hommes, Ro pour les deux sexes à Rewa) et par des codes de comportement,

- et ceux qui sont en plus choisis pour être chefs d'un mataqali, yavusa, tikina, vanua ou matanitu ${ }^{4}$.

À l'échelle locale, le pouvoir des chefs s'exerce en parallèle de celui de l'État, représenté par le Roko Tui et le bureau provincial (Provincial Office), et des autorités religieuses ou lotu ${ }^{5}$. Les attributions des chefs et la façon de les exercer dépendent notamment du rang de leur clan d'origine, de leur position plus ou moins forte au sein de ce clan (détention ou non d'un titre, rang du tokatoka dans lequel on est né, qualité de l'ascendance maternelle, position d'aîné ou de cadet, genre, etc.) et des spécificités culturelles

1. Fidji a vu arriver à partir de 1879 plusieurs vagues de migrants originaires d'Inde. La communauté d'origine indienne représentait 37,5\% de la population totale en 2007 et dépassait même la communauté mélanésienne lors des recensements de 1946, 1956, 1966, 1976 et 1986, la tendance s'inversant à partir de 1996 (Fiji Islands Bureau of Statistics, 2010). Par convention, je nommerai «Indo-Fidjiens » les personnes issues de l'émigration indienne et " Fidjiens » les Mélanésiens. Les relations des deux communautés ont été marquées par de fortes tensions et la victoire aux élections générales de partis soutenus par les Indo-Fidjiens fut l'un des éléments déclencheurs des coups d'État de mai 1987 et mai 2000 (Fraenkel, 2007 : 422). La politique de construction nationale " à marche forcée » engagée par le gouvernement issu du quatrième coup d'État de l'histoire du pays (survenu le 5 décembre 2006) s'est accompagnée entre autres de l'utilisation du mot «Fidjien » pour les deux communautés ethniques, la population mélanésienne étant désormais officiellement désignée par l'expression iTaukei (autochtone).

2. Il faut fortement souligner que le système politique dit traditionnel ou coutumier s'est en partie constitué dans le courant du XIX ${ }^{e}$ siècle et après la cession de Fidji à la Couronne britannique en 1874. L'un des principes directeurs de l'administration coloniale fut, en effet, que le pays devait être gouverné " au travers des institutions fidjiennes " (indirect rule). Ceci explique que l'autorité administrative locale ait été largement déléguée aux chefs autochtones, en même temps que leurs attributions étaient redéfinies par le pouvoir central (Scarr, 1970 ; MacNaught, 1974). La création d'un grand conseil des chefs (1876) et la division de Fidji en trois confédérations de chefferies (matanitu), placée chacune sous l'autorité d'un chef suprême, remontent, par ailleurs, à la période coloniale.

3. On peut dire très rapidement que le mot vanua désigne à la fois un territoire et une population se référant à un même chef (par exemple : le vanua de Rewa, le vanua de Nabukebuke...). L'appartenance à un vanua contribue à définir l'identité personnelle et implique entre autres des obligations coutumières vis-à-vis du chef et de sa famille (prestations, étiquette), et des relations cérémonielles avec d'autres clans répartis dans les différents villages (koro). L'association de plusieurs vanua sous l'autorité d'un chef constitue un matanitu, le terme s'appliquant aujourd'hui aux trois grandes confédérations de Kubuna (sous l'autorité du Vunivalu Tui Kaba de Bau), de Burebasaga (sous l'autorité du Roko Tui Dreketi de Rewa), et de Tovata (sous l'autorité du Tui Cakau de Taveuni). Matanitu est aussi l'expression désignant le gouvernement et l'État moderne. Vanua, qui est traduit littéralement par le mot " terre " (land), peut désigner le peuple non noble par opposition aux chefs (turaga), et également, dans certains contextes, l'organisation sociopolitique coutumière ( $c f$. Tomlinson, 2002, pour une exposition de la complexité sémantique du mot vanua). Le mot tikina, enfin, désigne une partie du vanua et un district à l'intérieur de l'unité administrative que forme la province (yasana).

4. Mataqali est le plus souvent traduit par "clan » ou "sous-clan » en anglais. À Rewa et dans d'autres régions de Fidji, les mataqali se composent de plusieurs tokatoka (famille étendue), plusieurs mataqali hiérarchisés formant une yavusa (traduit en général par le mot " tribu »), la plus vaste unité de l'organisation politico-familiale. Un individu appartient généralement aux tokatoka, mataqali et yavusa de son père, mais des variantes " matrilinéaires » et d'autres concernant la hiérarchie et les dénominations des unités familiales ont été décrites à Fidji (Walter, 1978 : 353 ; Ward et Kingdon, 1995 : 202).

5. Le mot lotu se réfere au christianisme au sens large, mais désigne aussi le méthodisme dans certains contextes (Newland, 2009 : 189). Le méthodisme est aujourd’hui la religion dominante à la fois à Fidji et chez les Mélanésiens. On dénombrait 66,5 \% de Fidjiens d'obédience méthodiste lors du recensement de 1996 (ibid. : 187). 
des différentes provinces. À Rewa, les habitants considèrent que les détenteurs de titres doivent à la fois contribuer à élever le statut de leur communauté et à construire la prospérité et l'unité sociale, notamment par une activité de médiation des conflits, par des alliances prestigieuses et utiles, par l'accomplissement d'obligations cérémonielles : participation au cercle local du yaqona $a^{6}$, échanges de prestations cérémonielles avec leurs sujets et les autres chefferies, etc. Notons que les chefs fidjiens sont comme dans d'autres pays du Pacifique associés à une forme de pouvoir surnaturel ou mana, qu'ils possèdent en plus ou moins grande quantité, et qui est reconnu par ses manifestations dans le monde, notamment en termes de succès et d'efficacité. Dans la province de Rewa, l'autorité des chefs dûment installés, assise sur une décision collégiale interne au mataqali et sur une généalogie qui les relie aux ancêtres (même si des contestations plus ou moins fortes, voire des changements d'allégeance, sont encore possibles) précède toujours par principe celle des agents locaux du gouvernement. En plus de l'autorité qu'ils ont à l'échelle locale sur leurs sujets, les membres de l'élite noble sont intimement associés aux affaires nationales au travers d'une institution créée en 1876 par le pouvoir colonial, le Bose Levu Vakaturaga ou Grand Conseil des Chefs (Great Council of Chiefs), chargé entre autres de la nomination du président et du viceprésident (Newbury, 2006). Depuis l'indépendance, obtenue en 1970 par Ratu Sir Kamisese Mara, grand chef des îles Lau, les chefs ont aussi régulièrement occupé les plus hautes fonctions gouvernementales et formé ou soutenu des partis politiques puissants. Au bout du compte, la participation des chefs à la vie nationale semble autant le fruit d'une politique coloniale ('Angleterre avait besoin de l'influence individuelle des chefs et de les unir artificiellement pour bâtir la colonie) que d'un héritage culturel leur donnant forcément un rôle moteur au sommet de la société.

Depuis plusieurs années, le rôle national des chefs et même les bases plus générales du système politique coutumier ont été au cœur d'un débat relayé par les médias et impliquant les deux grands groupes ethniques. Alors que les chefs étaient vus depuis le $\mathrm{xIX}^{\mathrm{e}}$ siècle comme les meilleurs garants des intérêts fidjiens et comme des participants logiques au leadership national, des commentateurs ont commencé de pointer, de façon de plus en plus régulière, l'existence d'intérêts particuliers au sein du grand conseil des chefs (effet de sa politisation à partir des an- nées 1980 et des rivalités statutaires entre chefs et familles de chefs), le fait que certains dignitaires aient pu œuvrer " contre l'unité nationale " en entrant en sympathie avec des mouvements ethnonationalistes ${ }^{7}$, voire le caractère anachronique d'un système favorisant l'origine familiale et les relations d'allégeance plutôt que les capacités personnelles. Dans ce contexte, des voix se sont élevées en faveur d'une plus grande séparation des sphères politiques " coutumière " et " démocratique ", voire pour une dissolution du grand conseil des chefs (cf. Yabaki, 2006 ; Fiji Sun, 14/04/2007 a et b). Le rôle national des chefs est aussi devenu l'une des cibles du gouvernement amené au pouvoir par un coup d'État début décembre 2006. Pour son principal auteur, le contre-amiral Frank Bainimarama, l'édification nationale tenue en échec depuis l'indépendance impliquait de limiter l'influence du grand conseil des chefs, dominé par des dignitaires des provinces de l'Est fortement impliqués dans les partis pro-mélanésiens. Lorsqu'en avril 2007 le grand conseil des chefs s'opposa ouvertement au régime en refusant d'entériner le choix de Ratu Epeli Nailatikau comme vice-président, la sanction fut immédiate et le conseil interdit de siéger jusqu'à nouvel ordre. Une commission fut aussi mise en place pour réviser ses attributions. Les recommandations de cette commission, impliquant notamment un accroissement de l'emprise du gouvernement sur le grand conseil des chefs, furent rendues publiques en février 2008 (Norton, 2009 : 112). Toutefois, le conseil ne fut pas pour autant ré-autorisé à siéger. Ces mesures totalement inédites ont soulevé l'inquiétude chez une partie des Fidjiens qui y ont vu une mise en danger de l'identité autochtone, aggravée par des propos considérés comme irrespectueux à l'égard des chefs ("If they dismantle and destroy the institution [le grand conseil des chefs], they destroy Fijian people " Fiji Times, 14/04/2007).

En définitive, l'opinion mélanésienne semble traversée aujourd'hui par deux tendances : d'une part, l'affirmation d'une importance de la tradition, dont le système des chefferies serait un aspect central (et dont les chefs seraient aussi des protecteurs au sein du grand conseil des chefs) et d'autre part, une interrogation de fond sur les attributions des chefs, assortie d'une demande de modernisation. D'après $S$. Ratuva :

"In fact some Fijians see the Great Council of Chiefs as a liability to Fijian progress while some see it as an important vehicle for chiefly power. Increasingly chiefly authority is being questioned by a lot of new generation Fijians. "(Turaga, 05/2007: 24)

6. Le yaqona ou kava est une boisson légèrement narcotique, préparée à partir des racines du Piper methysticum. De très nombreuses cérémonies impliquent l'offrande et la consommation de yaqona. La distribution des coupes suit toujours un ordre précis et met en valeur le rôle cérémoniel et les relations statutaires des participants.

7. Dont l'idéologie est associée aux coups d'État de mai et septembre 1987 et de mai 2000 (Firth et Fraenkel, 2007 ; Fiji Times, 01/09/2004). 
L'attachement au principe d'un pouvoir fort des chefs, de même que la définition de la tradition et des changements souhaitables, varient considérablement d'une province à l'autre et au sein de chacune d'entre elles. Cette variabilité s'explique entre autres par celle des organisations sociales dans l'archipel fidjien', par le rôle historique joué par les différentes régions ${ }^{10}$, par la diversité de leurs situations économiques, par les intérêts politiques et les alliances de leurs leaders. La place à donner aux chefs est ainsi devenue à Fidji, comme ailleurs dans le Pacifique, un enjeu majeur de la construction nationale, en même temps qu'un motif de désaccord et de division dans la population (particulièrement, mais pas uniquement, dans la communauté mélanésienne) et de façon encore accrue depuis la suspension du grand conseil des chefs. Depuis la survenue du coup d'État en décembre 2006, les chefs eux-mêmes, loin de faire bloc, se sont progressivement séparés en deux camps rivaux. Un premier camp rassemble des chefs surtout originaires de l'Est de Viti Levu et de Vanua Levu, hostiles au régime en place, et notamment plusieurs "grands chefs " (paramount chiefs) détenteurs de titres majeurs. Un second camp rassemble les chefs partisans du gouvernement, qui ont éventuellement, contre ce que prescrit la coutume, fait sécession avec leurs supérieurs hiérarchiques ennemis du régime.

Dans ce contexte, cet article examinera les représentations populaires du système politique coutumier, et en particulier des rôles locaux et nationaux des chefs, au sein d'une chefferie fidjienne. Il s'appuie sur des enquêtes conduites entre décembre 2007 et février 2011 dans la province de Rewa, située dans le Sud-Est de la grande île de Viti Levu, et réputée pour son organisation politique très centralisée et fondée sur un pouvoir fort des chefs ${ }^{11}$. Les questions que je pose sont les suivantes : existe-t-il dans cette province réputée conservatrice une forme de critique ou de remise en cause des leaders coutumiers? Si c'est le cas, quelles prérogatives ou réalisations des chefs sont mises en cause par les habitants, et retrouve t-on par exemple les arguments du contre-amiral Bainimarama ayant servi à justifier la suspension du grand conseil des chefs (Fiji Government Online Portal, 12/04/2007) ? Pourquoi les chefs peuventils a contrario être considérés comme essentiels à l'équilibre du vanua ou de la nation ? Quelle différence est faite entre les chefs locaux et les chefs au sens large, ou encore entre les nobles comme classe sociale et les porteurs de titres? Quelles divergences d'opinion apparaissent chez les habitants et quels enjeux locaux cette diversité peut-elle recouvrir ? D’une façon générale, quels sont les facteurs qui contribuent aujourd'hui à transformer les principes de construction et de reconnaissance du pouvoir des chefs ? Je vais d'abord procéder à une présentation de Rewa et de son organisation politique, avant de revenir, dans une deuxième partie, sur la perception et les métamorphoses du pouvoir des chefs à l'intérieur de la province.

\section{"The most perfect example of a Fijian State known to us " 12}

\section{L'organisation sociopolitique de Rewa}

Rewa est avec ses $272 \mathrm{~km}^{2}$ la plus petite des quatorze provinces que compte Fidji. Située dans la partie orientale de la grande île de Viti Levu, son territoire se distribue sur deux zones non contiguës séparées par la province de Naitasiri, auxquelles s'ajoutent encore l'île de Beqa et ses deux districts de Sawau et Raviravi. Rewa se compose de neuf districts (tikina) hiérarchisés, dont le plus important est celui de Rewa, dans le delta de la rivière Rewa (au Nord-Est de Suva, la capitale du pays). Le village principal du tikina de Rewa, Lomanikoro, est à la fois le centre administratif de la province moderne et la résidence du chef coutumier du

8. Si c'est généralement le rôle national des chefs qui est pris pour cible, ou encore leur influence sur l'administration des provinces, un commentateur anonyme évoque pour sa part une " rébellion populaire naissante " contre l'autorité des chefs coutumiers: "This indicates a nascent grass roots rebellion that is pretty hard to stop once it takes root. That's what the chiefs are worried about" (publié sur internet: Fiji: the way it was, is and can be, 19/11/2009, http://crosbiew.blogspot.com/).

9. Les régions de la partie Est de Fidji montrent depuis le xixe siècle des organisations sociopolitiques plus pyramidales et fondées sur un pouvoir fort des chefs que les régions intérieures et l'Ouest du pays (Derrick, 2001 : 6; Brison, $2001: 318$ ).

10. Les chefs des grands royaumes de l'Est (Lau, Bau, Rewa, Cakaudrove) ont constamment dominé la scène politique nationale depuis la période coloniale et encore après l'indépendance, générant des frustrations et le sentiment d'un " colonialisme interne " dans la partie Ouest, où se trouve aussi la majorité des ressources économiques du pays : aéroport international, activité touristique, canne à sucre, etc. (Bose et Fraenkel, $2007: 225)$.

11. Cet article est basé sur des enquêtes conduites pendant dix-sept mois à Rewa, entre décembre 2007 et février 2011, principalement à Lomanikoro mais aussi dans d'autres villages et districts. Les recherches ont été financées en 2008 et 2009 par une bourse post-doctorale de la Fondation Singer-Polignac, puis par le programme collectif «Logiques locales, logiques nationales : mutations politiques dans trois pays dits mélanésiens" (LocaNatPol) soutenu par l'ANR. J'ai volontairement omis de préciser les noms des personnes ayant partagé leurs opinions ou connaissances sur le système des chefferies, sujet considéré comme sensible, surtout si l'on évoque sa propre province, en me limitant de fait à des indications générales.

12. Cette déclaration apparait dans un rapport de la Native Lands Commission consacré à la tenure foncière de Rewa en 1893 (ANF, Colonial Secretary Office, Minute Paper n³163/1893, p. 14). 
vanua, qui porte le titre de Roko Tui Dreketi. Outre l'éclatement géographique, une des $\mathrm{Ca}-$ ractéristiques de la province est la pauvreté de ses ressources naturelles, avec une majorité de terres marécageuses difficiles à mettre en valeur, Rewa étant en plus très exposée aux inondations dans la zone basse du delta et sur les côtes (Raj, 2004). La province n'en est pas moins densément peuplée depuis l'époque précoloniale ${ }^{13}$, grâce notamment à la culture du taro géant, ou via, espèce qui supporte une immersion prolongée pendant les inondations de l'été austral. Rewa est par ailleurs la province dominante de la confédération (matanitu) de Burebasaga, la plus vaste des trois que compte le pays, et la seconde après Kubuna en termes d'importance hiérarchique. Ceci implique que dans les rituels, le chef de Kubuna boit le yaqona avant celui de Burebasaga, Tovata prenant la troisième coupe. Burebasaga regroupe outre Rewa les provinces de Kadavu, Nadroga, Navosa, Serua, Namosi, et une partie de Ba. Rewa est surtout connue pour avoir été engagée entre 1843 et 1855 dans une longue série de guerres qui l'ont opposée au royaume de Bau et qui ont fini par impliquer, sous l'effet des alliances, des conquêtes, et des relations de parenté, une grande partie de l'archipel fidjien (Sahlins, 1991, 2004). Ces guerres, sur lesquelles existe une littérature exceptionnellement détaillée, ont été finalement remportées par Bau, avec l'appui du roi George Tupou de Tonga et d'Européens (Sahlins, $2004: 15,100$ ). Elles ont modelé la hiérarchie sociopolitique qui fait toujours référence aujourd'hui, avec la domination du matanitu de Kubuna et de son chef, le Vunivalu Tui Kaba, pour lequel fut créé le titre suprême de Tui Viti (roi de Fidji). Depuis 1989 , des conflits interfamiliaux ont empêché l'élection d'un nouveau Vunivalu Tui Kaba à Bau, situation qui a favorisé le rayonnement de Rewa en donnant de facto une préséance cérémonielle au Roko Tui Dreketi.

La province moderne (yasana) épouse les contours du territoire coutumier des chefs de Rewa, les différents tikina ou villages ayant été agrégés, à des époques plus ou moins anciennes, à l'issue de conquêtes ou d'alliances. Cette histoire contribue à définir leur rôle cérémoniel, notamment les types de services (i tavi : rôle spécifique, devoirs) rendus à la
Grande Maison (vale levu, le clan du chef suprême), et aussi leur position plus ou moins élevée dans la hiérarchie locale. Les remaniements administratifs de la période coloniale ont toutefois amputé le royaume de villages et de districts qui lui étaient autrefois associés, comme Tokatoka (autrefois bati du Roko Tui Dreketi), à présent dans la province de Tailevu. Lorganisation politique en vigueur à Rewa et dans d'autres régions de Fidji (Sahlins, 2004 : $61,64)$ implique une dyarchie composée d'un chef sacré et d'un chef de guerre, ou Vunivalu ("racine de la guerre »), de rang légèrement inférieur. À Rewa, l'ensemble des clans et des villages sont répartis entre ces deux chefs ${ }^{14}$, mais tous reconnaissent en théorie la supériorité statutaire du Roko Tui Dreketi, ou chef suprême. Les résidences du Roko Tui Dreketi et du Vunivalu se font face au cœur du village-capitale de Lomanikoro, et les autres clans et villages se distribuent, à partir de cet espace central, en fonction de leur importance hiérarchique et des types de services rendus à la chefferie. À Lomanikoro même, à côté des clans nobles de Valelevu et Nukunitabua, les différents mataqali se définissent comme hérauts (matanivanua), conseillers et "faiseurs de chefs " (sau turaga $)^{15}$, prêtres (bete), guerriers (bati), ou servants (qase ni vale). À proximité immédiate de Lomanikoro se trouvent les villages de $\mathrm{Na}$ bua et de Sigatoka, habités par deux groupes d'origine tongienne, représentés comme des «favoris » de la maison royale et anciennement spécialistes de la navigation. Dans ce proche périmètre vivaient aussi les charpentiers ( $m a-$ taisau) de Dorokavu, dont les mauvaises terres, trop exposées aux crues, ont été récemment abandonnées pour une position plus sécurisée sur la rive opposée. Toujours dans le tikina de Rewa, Nasilai et Nukui sont des villages kai wai, de "gens de l'eau ", fournissant pour le premier des tortues marines, pour le second du poisson à la Grande Maison. Les six autres villages du tikina sont habités par une seule yavusa, la Burenivalu, dont les membres, dit kai Rewa (gens de Rewa), seraient les habitants originels de la province. Les membres de la Burenivalu sont réputés avoir attiré et installé le premier Roko Tui Dreketi et entretiennent avec ces chefs des relations ambivalentes, im-

13. Le père Jean-Baptiste Bréhéret écrit en 1861 à propos de Rewa : «C’est la partie la plus peuplée de Fidji, celle que les [pères] Favier et Leberre ont maintenant à évangéliser. Dire que la population est de cinquante mille âmes n'est, je crois, pas assez " (lettre du 20/09/1861, APM, 1383/19674).

14. Chaque mataqali, yavusa ou village a un lien cérémoniel privilégié soit avec le Roko Tui Dreketi, soit avec le Vunivalu. Dans le tikina (district) de Rewa par exemple, six des douze villages se réferent au Roko Tui Dreketi, et six au Vunivalu. Cette situation n'empêche pas de reconnaître l'autre chef, et d'être lié à lui par des obligations cérémonielles. Cette bipartition dont l'origine n'est pas claire (d'après un villageois de Lomanikoro, un ancien Roko Tui Dreketi aurait demandé à une partie de ses sujets de se mettre au service du Vunivalu, pour l'honorer) fut déjà observée par A. Hocart entre 1912 et 1913: « They [le Roko Tui Dreketi et le Vunivalu] divide Rewa and all subject villages and carpenters between them. There is not a village in which they have not both got authority" ( $\mathrm{ms}: 431$ ).

15. Le clan des sau turaga joue un rôle dans la validation du choix du Roko Tui Dreketi (infra). 
pliquant la soumission, mais aussi des prérogatives qui les distinguent des autres habitants ${ }^{16}$, ainsi qu'une forme de contrôle sur la continuité de la lignée (infra). Les autres districts et villages de la province sont aussi liés au Roko Tui Dreketi et au Vunivalu par des devoirs cérémoniels de différentes natures. La littérature européenne et la tradition orale suggèrent que les villages ou tikina avaient autrefois des statuts très variables, oscillant entre une situation d'assujettissement les soumettant au tribut, et les désignant comme pourvoyeurs de victimes humaines pour les sacrifices, et de simples alliés militaires (comme, par exemple, le district de Noco).

Les rôles spécifiques des différents clans, villages et districts restent largement reconnus, bien que s'exprimant surtout désormais à l'intérieur des rituels. Les fêtes familiales des chefs (mariages, décès, levées de deuils, présentation d'enfants aux parents maternels, etc. $c f$. Drova Tadrau, 1985 : 70), les cérémonies d'accueil de chefs étrangers, mais aussi les réunions du conseil provincial ${ }^{17}$, donnent l'occasion de faire apparaître ces fonctions sociales, leur interdépendance, ainsi que la hiérarchie des mataqali et des tikina, organisée en référence au chef suprême. Les funérailles du Roko Tui Dreketi Ro Lalabalavu Mara en juillet 2004 ont ainsi mobilisé l'organisation sociale de Rewa avec une précision et à une échelle exceptionnelles, en mettant notamment en valeur le rôle des bati (guerriers) de Toga et Vutia, chargés de protéger le corps de la défunte et les tabous hiérarchiques. Létiquette qui organise les relations chef/sujets, et d'une façon moins prononcée celles des membres des clans nobles (turaga) et du peuple (également nommé vanua, "la Terre »), reste aussi une réalité quotidienne, qui a fait désigner Rewa comme l'une des provinces les plus conservatrices de Fidji pour ce qui a trait au " respect de la hiérarchie ". Ces rôles mais aussi la manière dont ils sont répartis entre les clans ont évolué depuis les débuts de la période coloniale, à la fois pour adhérer à des besoins nouveaux et sous l'effet des relations politiques changeantes et éventuellement conflictuelles au sein du vanua. Par exemple, la fonction prestigieuse de héraut principal du Roko Tui Dreketi a été assumée par différents mataqali. Dans tous les cas, la cohésion du vanua de Rewa repose en premier lieu sur la référence commune à un chef suprême, le Roko Tui Dreketi, autour duquel les différents clans et leurs propres titres familiaux se distribuent harmonieusement, " comme des planètes autour du soleil " (" like planets around the sun", selon une formule d'A. Hocart que reprend M. Sahlins au sujet du Roko Tui Dreketi. 2004 : 61). Les sujets du vanua ayant la responsabilité collective de faire exister et d' "élever » le statut du chef par leur déférence (vakarokoroko) et leur activité cérémonielle. Il est possible que l'asymétrie caractérisant les relations du Vunivalu et du Roko Tui Dreketi ait été précédée par un dualisme plus prononcé, qui se rappellerait dans les termes d'adresse accompagnant les activités rituelles ${ }^{18}$ et que suggère peut-être l'organisation spatiale du centre de Lomanikoro, où les deux résidences sont mitoyennes (Sahlins, ibid). La situation contemporaine montre toutefois une nette prédominance du chef sacré, renforcée par son statut de chef suprême du vaste matanitu Burebasaga (alors que le statut du Vunivalu n'est reconnu qu'à Rewa), qui offre une sorte de reflet inversé de la situation de Tailevu, dominée par le chef de guerre ou Vunivalu Tui Kaba. La relation n'en a pas moins été marquée pendant la période coloniale par des tensions et par une compétition active pour le leadership local, dans laquelle les autorités britanniques (qui octroyaient les postes administratifs locaux et les pouvoirs associés à leurs meilleurs alliés) ont joué un rôle notable.

En plus d'être le village-capitale des chefs de Rewa, Lomanikoro est aussi le cour administratif et politique de la province : à la fois siège du bureau provincial (chargé de l'administration et de la trésorerie), lieu de réunion du conseil provincial, et lieu de résidence du Roko Tui Rewa, le chef exécutif du conseil provincial. De fait dès les années 1870 , les postes de fonctionnaires chargés de l'administration locale ont été régulièrement attribués à des chefs éminents de Rewa, dont l'autorité devait faciliter le contrôle des populations et l'application des décisions des pouvoirs coloniaux. Cette politique a favorisé une certaine identification de l'unité administrative qu'est la province, théoriquement sous contrôle du gouvernement, et du vanua (chefferie), régi par des règles totalement différentes. Le premier Roko Tui Rewa de l'histoire fut ainsi logiquement le Roko Tui Dreketi Ro Rabici (nommé en 1874), secondé, à la tâche de Buli ${ }^{19}$, par le Vunivalu Ro

16. Les membres de la yavusa Burenivalu sont décrits à Lomanikoro comme des "servants ", attachés à la maison du chef suprême et chargés notamment de cuisiner sa nourriture. Ils sont aussi les seuls habitants de Rewa à ne pas être affectés par l'influence surnaturelle du Roko Tui Dreketi, ils peuvent manger les restes de sa nourriture, le masser (" even step on him " : marcher sur son dos) et sont responsables d'enterrer son corps. Les observations d'A. M. Hocart au début du xx ${ }^{\mathrm{e}}$ siècle montrent là encore que ce rôle a une certaine antiquité : "Of the surrounding villages Nadoi is the leader of the Rewans, the original inhabitants. The word of the Noble Lord of Dreketi comes direct to them (i. e. not through an envoy). They are his own men and bring food to him [...] The men of Nadoi are the hand of the holy thing' [...] they dress the dead chief, and bury him" (Hocart, ms : 433). 17. Provincial Council, conseil administrant la province, sous l'autorité du Fijian Affairs Board.

18. On rend hommage aux deux chefs (qu'ils soient présents ou pas) avant tout discours, toute présentation d'objets cérémoniels.

19. Le Buli (une fonction aujourd'hui disparue) était le chef administratif d'un district, à l'intérieur de la province placée sous l'autorité du Roko (Nayacakalou, 1985 : 83). 
Veceli Namusudroka. Le fils de Ro Rabici, Ro Lutunauga Tuisawau qui prit sa succession en tant que Roko Tui Dreketi, occupa alternativement les offices de Buli et de Roko Tui Rewa, mais son insoumission et ses frasques amoureuses (qui un temps semblent avoir menacé la paix entre Bau et Rewa) devaient conduire à sa destitution en juin 1903 et à son remplacement par le très influent Ro Joni Mataitini, du mataqali du Vunivalu (ANF, Colonial Secretary Office, Minute Papers n²697/1903, $\left.\mathrm{n}^{\circ} 1662 / 1904, \mathrm{n}^{\circ} 3376 / 1913\right)$. La vie de la province fut marquée dans ce cadre et jusqu'à nos jours par de nombreux scandales financiers, où des chefs se voyaient accusés d'utiliser la manne des fonds publiques comme une ressource personnelle, en quelque sorte coextensive de leur statut et de leurs prérogatives au sein du vanua. Une imbrication des deux systèmes politiques se fait toujours sentir à de multiples niveaux, avec par exemple une attribution systématique de la position de président du conseil provincial au Roko Tui Dreketi ou au Vunivalu et l'attribution d'autres rôles, comme celui de vice-président, selon des critères faisant intervenir le clan d'origine et la possibilité rituelle de s'adresser directement au chef suprême ${ }^{20}$. Les membres des clans nobles de Rewa ont par ailleurs été fortement associés à la vie politique nationale, à la fois au travers du grand conseil des chefs et en tant que parlementaires, ou militants et fondateurs de partis politiques majeurs après l'indépendance. Toutefois, les Roko Tui Dreketi ont été traditionnellement en retrait de la vie politique nationale après avoir accédé à leur titre, jusquau règne de Ro Teimumu Kepa, dont il sera largement question ici.

L'absence d'une règle fixe de succession et la multiplication des épouses ont favorisé d'intenses rivalités au sein de la famille régnante, mais il n'y a pas eu de vacance prolongée du pouvoir à partir du règne du Roko Tui Dreketi Ro Rabici, qui succéda à Ro Qaraniqio, décédé en janvier 1855 (Sahlins, 2004 : 165 ; lettre du père Mathieu, 10 mars 1855, APM, 1083/19672). Cette continuité, même si les choix des divers Roko Tui Dreketi ont pu être accompagnés de contestations (émanant notamment des enfants d'épouses non légitimes nés avant le chef suprême), a favorisé à la fois la cohésion interne de la province, son prestige extérieur et sa capacité d'influence au travers du grand conseil des chefs; notamment après le décès du dernier Vunivalu Tui Kaba en 1989, et en 2004 du puissant Tui Nayau des îles Lau, qui n’a pas non plus été remplacé. Cette continuité a en outre été facilitée à la fois par l'absence de cérémonie d'installation pour les Roko Tui Dreketi et par la possibilité, rare à Fidji, de transmettre le titre suprême à des femmes. De fait, en l'absence d'héritier mâle parmi les enfants du Roko Tui Dreketi Ro Joji Cokanauto Tuisawau et de Adi Asenaca Vosailagi Makutu son épouse légitime, le titre est revenu en 1961 à leur fille aînée Ro Lalabalavu Mara, puis en 2004 à sa cadette Ro Teimumu Kepa. Rewa a donné dès le $\mathrm{XIX}^{\mathrm{e}}$ siècle l'image d'une province où le pouvoir central était spécialement fort, avec des chefs tenus dans un très haut respect et que l'administration coloniale eut souvent le plus grand mal à garder sous contrôle. Aujourd'hui, l'autorité du Roko Tui Dreketi transparait dans le respect de l'étiquette et des devoirs cérémoniels (visible par exemple au moment des préparatifs du Bose Ko Viti en 2008-2009 ${ }^{21}$ ), et dans la relative cohésion du mataqali Valelevu et du Bose ni Vanua ${ }^{22}$, quand la chefferie de Bau se montre très divisée jusqu'au sommet. Les enquêtes conduites entre fin 2007 et 2011 montrent néanmoins que le pouvoir des chefs fait aussi débat dans ce « bastion de la hiérarchie ", et que l'attachement au système des chefferies peut ou a pu recouvrir des insatisfactions. D'une façon générale, les doutes ou mécontentements exprimés ne s'inscrivent pas dans l'esprit d'une révolution moderniste, d'une remise en cause de fond des différences statutaires entre le peuple et les chefs. La dénonciation d'un anachronisme ou d'une injustice fondamentale du système politique coutumier, qui apparaît dans les médias et dans certains milieux intellectuels, n'a pratiquement aucune réalité sur le terrain à Rewa. En revanche, les critiques visent en priorité la manière dont les chefs, soit à l'échelle locale, soit à celle du pays, honorent des responsabilités venant en complément et en contrepartie de celles du peuple. La définition offerte de ces responsabilités diffère selon le statut du chef (chef local ou étranger, détention ou non d'un titre, qualité de ce titre). Elle est aussi influencée par le rôle traditionnel des informateurs, par leurs éventuelles relations de parenté avec les chefs, par leurs convictions politiques, par le type d'éducation qu'ils ont reçu, et peut varier selon le contexte ou à l'intérieur de stratégies personnelles et collectives. On constate ainsi que tous les habitants n'ont pas la même vision des rapports à avoir avec la scène politique nationale, de la juste manière pour les chefs de s'inscrire dans la tradition et dans la modernité, ou la même interprétation des ri-

20. Communication personnelle du vice-président du conseil provincial de Rewa, originaire du district de Vutia.

21. Le Bose Ko Viti est un rassemblement religieux méthodiste organisé normalement tous les deux ans. Rewa devait accueillir cette manifestation en août 2009. À la demande du chef suprême, les différents tikina et villages ont entrepris de réunir l'argent et les grandes quantités de nattes, racines de yaqona, porcs... nécessaires pour l'accueil des visiteurs et les échanges (solevu). La manifestation fut finalement annulée par les autorités militaires, qui considérèrent qu'elle dissimulait des objectifs politiques et des velléités de déstabilisation du régime.

22. L'assemblée des chefs des différents tikina de Rewa. 
valités entre chefs et familles de chefs. La partie suivante examinera d'abord le discours populaire portant spécifiquement sur les chefs de Rewa, en mettant en évidence les critiques formulées et les approches variables de leurs rôles locaux et nationaux. Je m'intéresserai ensuite aux représentations du grand conseil des chefs et du système des chefferies au sens large.

\section{Comment être chef ? Les représentations du pouvoir coutumier à Rewa}

Le Roko Tui Dreketi : trop précieux pour la politique?

En succédant à Ro Lalabalavu Mara en 2004, Ro Teimumu Kepa a reproduit un changement majeur apporté par sa sœur aînée : pour la seconde fois, une femme catholique devenait Roko Tui Dreketi, quand ces chefs avaient toujours été des hommes, et alors que le méthodisme est depuis longtemps la religion dominante dans les clans nobles de Rewa et de Fidji en général. Par la suite, le début du règne de Ro Teimumu Kepa a été placé à différents points de vue sous le signe de l'innovation. Il est d'abord admis que Ro Teimumu Kepa offre un profil plus " moderne " que sa sœur aînée, qu'elle se révèle plus accessible, moins attachée à l'étiquette et à la différenciation hiérarchique. Cette attitude qui contraste avec les coutumes locales de mise à distance, d'oisiveté cérémonielle, voire de mutisme du chef suprême, suscite des réactions ambivalentes. Avec, souvent chez les mêmes individus, un mélange de fierté, de gratitude, et de désapprobation. Les habitants d'une part louent l'ouverture d'esprit et l'éducation du Roko Tui Dreketi, sa compréhension fine des réalités sociales, et sa volonté de s'y adapter par un nouveau style de leadership. Toutefois l'assouplissement des tabous hiérarchiques est perçu aussi comme une forme de danger, pouvant ouvrir la voie à des comportements irrévérencieux, à une négligence par le peuple de ses devoirs traditionnels, et in fine à une dégradation du prestige et de la province et de son titre suprême. Le matanivanua et les proches collaborateurs du chef font dans cette mesure office de " gardiens du protocole ", et veillent au quotidien et en contexte cérémoniel à éviter le rapprochement ou l'identification de catégories qui, pour la bonne marche de la société, doivent être tenues fermement séparées. Par ailleurs, Ro Teimumu Kepa est le premier Roko Tui Dreketi à avoir tenté de concilier cette charge, à laquelle elle a accédé en septembre 2004, avec une carrière politique, sous la bannière du Soqosoqo Duavata Lewenivanua (SDL). Comme le disait un jeune chef :

"Roko Tui Dreketi the title never got involved with politics, it did get involved in some way, but not for everybody to see. " (Lomanikoro, février 2009)

En plus de son mandat de ministre de l'éducation du gouvernement de Laisenia Qarase, Ro Teimumu Kepa a obtenu en 2006 l'investiture du SDL pour briguer le siège de Rewa (communal seat) à la Chambre des Représentants. Cette situation qui soumettait le chef suprême au référendum populaire fut décrite comme une nouveauté radicale et comme une source de malaise pour la population de Rewa ${ }^{23}$. Un membre de l'équipe de campagne de Ro Teimumu Kepa soulignait la grande différence de sa situation au moment des élections générales de 2001, époque où Ro Lalabalavu Mara était encore Roko Tui Dreketi, et en 2006, c'est-à-dire après son accession au pouvoir. Ro Teimumu Kepa n'a plus de mandat national depuis le renversement du gouvernement Qarase en 2006, mais elle a continué d'exprimer son opinion sur l'actualité politique et les réformes en cours, en particulier sur la révision des attributions du grand conseil des chefs.

Une partie des habitants de Rewa, notamment dans les familles nobles, considère le choix du chef suprême comme une initiative certes audacieuse, mais finalement nécessaire. Pour ces informateurs, l'activité politique nationale serait le prolongement logique du rôle traditionnel des chefs : elle permettrait de conserver un vrai pouvoir d'action et une prééminence sociale globalement mis en danger par les changements de la société fidjienne (en somme de rester des chefs, dans un monde en mutation). Ro Teimumu Kepa pour sa part avait expliqué après les élections de 2006 que l'engagement politique était pour un chef une manière efficace d'agir pour le développement de son vanua:

"I have to be part of Government, I have to be in Cabinet because that is where all policies and decisionmaking is made at national level. And for us in Rewa, we do not have the resources so we have to look to Gov-

23. Un jeune chef décrit les paradoxes à résoudre au moment de la campagne, à Rewa, d'une candidate se trouvant être aussi le chef suprême de la province. Organiser meetings et débats, quand le protocole interdit de s'adresser directement au chef suprême (a fortiori de le critiquer publiquement), fut spécialement complexe. Il cite et commente les propos tenus à la candidate du sDL par un habitant: " Why is it [...] that the last time we ever saw you in this community hall was 2001? Only now that the election is here, you have chosen to come back. Where were you in these five years?' That was a very straightforward democratic question. To Ro Teimumu. They have the right. But to some of us who were sitting there... How can you speak like that to the Roko Tui Dreketi? She was wearing two hats sitting there. One was a democratic hat and one was the traditional hat. But it was a democratic process, that guy had every right to ask that question. Or any other person had any right to ask any question. But because of the other hat, that was overshadowing the democratic hat, that was the concern. [...] No, you cannot solve the conflict. We cannot solve the conflict. The person himself, or the Roko Tui Dreketi, she has to make sure which hat to wear " (Lomanikoro, février 2009). 
ernment for support. I cannot do that when I'm sitting in the village. " (Tudrau-Tamani, $2006: 15$ )

Des habitants ont aussi avancé que, dans une province peu touristique comme Rewa, où les chefs ne peuvent compter sur les revenus générés par la location de terrains aux hôtels, travailler (en parlant ici d'un mandat politique) est indispensable pour faire face aux obligations de la vie coutumière. C'est-à-dire, en particulier, pour pouvoir donner conformément à leur statut lors des échanges cérémoniels émaillant la vie du va$n u a$, et surtout dans les cérémonies impliquant d'autres chefferies : nourriture crue et cuite, yaqona, dents de cachalot ou tabua, nattes, tissu d'écorce peint (masi), et autres biens précieux.

"Everything concerns money. That's why she [Ro Teimumu Kepa] has to go to work. " (Lomanikoro, 2008)

Pour finir, des habitants ont émis l'idée que l'importance traditionnelle de Rewa devait avoir une traduction sur la scène nationale : qu'il était naturel que les vanua dominants sur la scène traditionnelle, et a fortiori ceux dont le chef suprême est bel et bien installé, soient aussi présents au gouvernement, représentés par leurs chefs ("They should be there. Bau should be up there and Rewa should be up there ").

Toutefois, un nombre supérieur d'habitants (en particulier, mais pas seulement, parmi les clans du vanua - c'est-à-dire non nobles) insistent $a$ contrario sur les conséquences fâcheuses d'une activité politique nationale du chef suprême, même si le renvoi du gouvernement en 2006 est aussi décrit comme une blessure et une humiliation collectives, et même si beaucoup soulignent que le chef a contribué au développement de Rewa dans ses différents mandats ("she puts development to the schools"). Pour commencer, pour une large partie des personnes, l'activité politique du chef suprême comporterait en soi un risque majeur : celui d'importer la division du monde politique national à l'intérieur du vanua. Les membres du vanua n'ayant pas la même sensibilité politique que le chef, pourraient, au final se détourner aussi du leader traditionnel dans le contexte local, ou au moins ne plus lui accorder un égal respect et une égale confiance. Le chef investi de son titre et colorant ce titre d'une préférence politique ne serait plus tout à fait capable, in fine, d'incarner et d'englober la totalité du vanua. Un autre point souvent évoqué est que, dans le cadre d'une fonction politique nationale, le chef suprême va presque forcément se trouver contredit, moqué, voire injurié par d'autres politiciens et adversaires politiques (en plus de se trouver soumis à l'autorité d'autres responsables, probablement issus de clans ou de provinces d'un rang moins élevé). De telles humiliations, décrites comme insupportables pour les sujets de
Rewa, porteraient atteinte à la dignité du titre et pourraient affecter par écho l'autorité traditionnelle du Roko Tui Dreketi. Dans le rôle de parlementaire ou de ministre, le Roko Tui Dreketi ne serait plus, en somme, protégé par les tabous hiérarchiques : le chef serait projeté au contraire dans un univers égalitaire et chaotique, fondé sur l'équivalence des droits et la libre parole, où son statut et la sacralité de son titre seraient constamment remis en cause. D'après un homme de Lomanikoro :

"In Parliament they speak out their mind. They say anything. Even if you are Roko Tui Dreketi they call you anything. They can call you a name. And at the same time it's in the medias People are looking and listening. They are looking at their high chiefs. Somebody from nowhere calling them... That's politics. This is not good." (juillet 2009)

Des habitants soulignent toutefois que cette argumentation d'ordre culturel a aussi pu devenir un prétexte commode, notamment chez les jeunes membres des familles nobles, pour forcer une répartition des tâches : au chef suprême les dignités du vanua, à ses proches parents, les responsabilités politiques et leurs vrais pouvoirs. En plus de ces outrages inévitables, la vie politique aurait comme autre conséquence négative d'éloigner le chef du territoire coutumier, en imposant de fréquents séjours, sinon une présence continue, dans la capitale. De fait, l'accession de Ro Teimumu Kepa au titre de Roko Tui Dreketi, alors qu'elle était encore ministre, a soulevé des inquiétudes dans une population qui, déjà, avait été longuement privée de la présence de Ro Lady Lalabalavu Mara. Ceci, en raison des mandats successifs de son époux Ratu Sir Kamisese Mara, qui fut Premier ministre après l'indépendance, puis président de Fidji de 1994 à 2000. D'une façon générale, l'éloignement géographique imposé par la politique priverait le vanua de l'influence spirituelle protectrice du chef, le sau ou mana associé au titre, en plus de permettre à d'autres forces d'occuper le terrain : les chefferies rivales, mais aussi les familles rivales à l'intérieur de la province. Ajoutons que le vanua est vu comme une fédération d'éléments hétérogènes, agrégés autrefois par la guerre ou les alliances, dont la cohésion dérive avant tout de la reconnaissance d'un même chef et des services cérémoniels accomplis pour ce dernier : en somme, le vanua de Rewa perd de sa raison d'être s'il est privé de sa " tête " et de ses cérémonies, il est structurellement menacé d'éclatement. Les habitants sont dans cette mesure uniformément reconnaissants à Ro Teimumu Kepa d'avoir choisi de s'installer à Lomanikoro, dans la résidence des Roko Tui Dreketi, d'où son mana irrigue l'ensemble du vanua. Les habitants comparent volontiers la situation de cette femme instruite, habituée à la vie urbaine, mais acceptant pour 
le bien commun de vivre au cour du vanua, et celle des nombreux chefs qui tentent d'exercer un contrôle à distance.

En définitive, une majorité d'habitants affirme désapprouver l'idée d'un engagement politique du chef suprême. Cette réticence existe aussi pour le Vunivalu, et pour d'autres chefs moins importants comme les turaga ni yavusa, mais de façon moins prononcée. Dans le cas $\mathrm{du}$ Vunivalu, il semble admis que la nature de son titre, tourné vers l'action et l'affrontement, est plus en accord avec une activité politique nationale, que son contact avec la scène politique est moins dommageable, moins teinté de danger que celui du chef sacré. Par ailleurs, les membres des clans nobles, classés comme turaga parce qu'ils sont nés dans un mataqali de haut statut, mais qui ne sont pas porteurs d'un titre, sont rarement critiqués pour leur activité politique, à moins que leur attitude porte atteinte à la réputation du mataqali et du vanua. La politique paraît même vue comme une orientation naturelle des jeunes membres des familles nobles, que leurs ambitions et leur goût du pouvoir pousseraient logiquement vers le " champ de bataille " national, en attendant, dans le meilleur des cas, de pouvoir succéder au titre familial. L'activité politique des chefs est souvent présentée néanmoins comme une source de division à l'intérieur des clans nobles et entre ceux-ci (une division qui prolonge et aggrave les rivalités existant pour le leadership traditionnel ${ }^{24}$ ), et, pour les chefs les plus importants, comme une menace à leur représentativité et à la sacralité de leurs titres. Elle exposerait qui plus est la population à des dilemmes et à des conflits d'allégeance : l'opinion politique personnelle a-t-elle droit de cité quand le chef d'un vanua est candidat aux élections ? Comment choisir quand plusieurs chefs s'opposent dans la compétition électorale ? Comment le prestige du perdant peut-il ne pas être affaibli, ou même sa légitimité locale menacée, quand tout le monde conclura à un manque de mana?

\section{Les rôles sociaux de la noblesse à Rewa}

Dans le mythe d'origine de la chefferie de Rewa, où l'on retrouve le schème de l'Étranger-Roi étudié par M. Sahlins (1985), le premier Roko Tui Dreketi est un cadet de haut rang nommé Ro Rawalai, arrivé de Verata, et pris au piège par les clans autochtones (yavusa Burenivalu) alors qu'il réside à Burebasaga. L'assimilation du jeune voyageur, dont la supériorité statutaire est immédiatement reconnue, ne s'effectue pas ici par le biais d'un mariage, mais par des dons de nourriture dont la qualité subjugue Ro Rawalai. Les hommes de la Burenivalu déclarent au jeune homme qu'il pourra goûter à l'envie les délicates rourou moci (feuilles de taro farcies de petites crevettes de rivière) et le crabe de mangrove (mana) s'il accepte de s'installer à Rewa. Ro Rawalai les suit alors sur la rive opposée, où il est transformé en chef local. Une forme de contrôle populaire s'exprime toujours aujourd'hui au moment d'élire le chef suprême : lorsque les membres du mataqali Valelevu se sont accordés sur le nom du successeur, ce choix doit être impérativement validé par le chef des sau turaga, qui dans ce contexte représente la population du vanua. Par ailleurs, pendant les périodes coloniale et postcoloniale, les membres de la yavusa Burenivalu sont plusieurs fois intervenus, soit pour empêcher la vacance du titre suprême $e^{25}$, soit pour prendre ouvertement parti pour un prétendant au titre jugé plus légitime qu'un autre. Il apparaît ainsi que les clans-sujets du vanua ont une responsabilité directe dans l'existence et la perpétuation de la dynastie des Roko Tui Dreketi, qui restent considérés cérémoniellement comme des cadets (gone turaga, enfant-chef), servis et nourris par les aînés (qase) de la yavusa Burenivalu. Une autre dimension, mise en valeur tant par les villageois non nobles que par les chefs, est que la relation chef/sujets se conçoit toujours comme un engagement réciproque : " a good 'give and take' relationship ". Si les clans de la Terre ont la responsabilité héritée d'accomplir certains types de tâches (services aux chefs, marques de respect, offrandes des premiers fruits...) qui représentent leur contribution personnelle à l'équilibre social, les turaga ont aussi leurs propres obligations et responsabilités. Chaque titre a toutefois ses spécificités, et la description offerte des devoirs des chefs tend à varier en fonction des individus et des clans, de leurs intérêts du moment, de leurs alliances. Par exemple, selon certains habitants de Lomanikoro, le Vunivalu aurait le devoir d'œuvrer à l'unification et à la prospérité du vanua, mais aussi de soutenir et protéger le Roko Tui Dreketi, en respectant constamment la distance hiérarchique entre les deux titres, quand d'autres mettent l'accent sur le parallélisme des deux chefs. Les membres des clans nobles qui ne possèdent pas de titres, même si leurs responsabilités sont moindres, sont censés honorer leur

24. B. Saumaki explique que ces rivalités du vanua ont des ramifications sur la scène politique nationale : "Historically in Rewa, the democratic process through the ballot box has indirectly been used as a means of bolstering the traditional legitimation of authority ». Il évoque la compétition pour l'investiture du SDL lors des élections générales de 2006, où s'opposèrent le Roko Tui Dreketi et son neveu Ro Vilive Tuisawau, anciennement prétendant au titre suprême (2007:217).

25. Au début des années 1920, une délégation de membres de la yavusa Burenivalu s'est rendue jusqu’à Tonga pour demander à Ro Emori Bikavanua Logavatu, fils de Ro Rabici, de prendre la succession après le décès de Ro Lutunauga Tuisawau. 
statut par un comportement approprié ${ }^{26}$ et travailler à l'unité de leur mataqali, notamment en soutenant le détenteur du titre.

À côté d'une attitude conforme à leur rang, les devoirs attribués par les habitants aux chefs de Rewa concernent en priorité la cohésion, la prospérité et le prestige du vanua, ou de leurs groupes familiaux respectifs. On attend invariablement des chefs, et plus que tout des porteurs de titres, qu'ils exercent un arbitrage des conflits, jouent le rôle de guides, attirent le succès et l'abondance par la grâce de leur mana, représentent dignement le groupe et son statut (par exemple en donnant avec largesse dans les cérémonies des autres chefferies), rassemblent et redistribuent des richesses : biens cérémoniels et nourriture pour les fêtes familiales, ou soutien financier aux projets collectifs, tels la construction d'une maison de réunion. Il semble admis qu'une profession valorisée (haute administration, médecine, enseignement) contribue positivement à la formation intellectuelle et au prestige personnel des chefs, même s'il est crucial qu'une telle activité n'empiète pas sur les missions locales du dignitaire. Tous les habitants ont aussi considéré que les chefs de Rewa devaient jouer un rôle central au sein du grand conseil des chefs, à la fois parce que le rang élevé de la province l'exige et parce qu'ils y représentent les intérêts de leur peuple face aux autres chefferies. Comme on a déjà pu l'apercevoir, l'exercice de fonctions politiques nationales, à l'intérieur du système politique dit " anglais " ou "démocratique ", n'est pas mis sur le même plan et fait par contre débat. Quand des habitants estiment que les offices nationaux doivent revenir aux chefs de haut rang, qui ont un droit et des aptitudes innés au commandement, d'autres estiment que les candidats idéaux sont les cadets des chefferies ayant reçu une solide formation intellectuelle (qui ont à la fois « la bonne généalogie et la crédibilité ", $c f$. Madraiwiwi, 2006 : 292) et n'ayant pas de hautes responsabilités dans le vanua. D'autres affirment enfin que des personnes non nobles peuvent assumer ces responsabilités avec tout autant de légitimité et d'efficience que les chefs, précisément parce que le leadership national s'inscrit dans une tradition politique étrangère, ne connaissant ni la hiérarchie des titres ni le protocole. Le fait que des personnes non nobles ou les cadets des chefferies puissent assumer des rôles nationaux, quand les principaux chefs se concentreraient sur le vanua, pose néanmoins d'épineux problèmes d'ordre statutaire. Un chef de rang secondaire accédant à un office public prééminent, par exemple un ministère, ne risque-t-il pas de faire de l'ombre à ses supérieurs hiérarchiques, ou même au chef suprême du vanua, en particulier parce qu'il ou elle aura des moyens financiers décuplés ? Avec le pouvoir économique de l'État, sa capacité à donner, à entretenir une clientèle, à drainer richesse et développement vers le vanua deviendra sans égale. Comme on l'a vu, des habitants estiment que c'est justement parce que la politique nationale peut (tout comme l'affairisme) aider à redistribuer les rôles à l'échelle locale, que les chefs les plus importants doivent y être engagés : pour que leur statut et les honneurs qui leur sont rendus ne deviennent pas l'équivalent d'une " coquille vide ", sans vraie capacité à transformer la société. Le désaccord avec la manière dont les chefs accomplissent leurs devoirs est manifesté selon des modalités codifiées ${ }^{27}$. La critique, même modérée, est exprimée la plupart du temps en présence d'une audience choisie et limitée, par exemple lors d'une conversation informelle autour du tanoa (le plat creux où la poudre de yaqona est mélangée à l'eau), et elle est malaisée en présence d'un étranger, tout particulièrement si la distance hiérarchique avec le chef en question est considérée comme grande. Dans le cadre des entretiens réalisés à Rewa, les habitants acceptaient généralement d'évoquer leurs doutes ou griefs, mais temporisaient en parlant du respect ressenti, en particulier, pour les deux principaux chefs ; une précaution moins fréquente quand les critiques concernaient les chefs d'autres provinces, même avec des titres élevés. De même, une erreur semble plus souvent attribuée à un groupe de personnes (entourage traditionnel d'un chef, ou son tokatoka), et au premier titre aux " mauvais conseillers " du chef, qu'au chef lui-même. Ces groupes étant souvent évoqués, qui plus est, de façon allusive, par exemple au travers de leur situation géographique dans le village : "this side" " " that side ". Par ailleurs, il est apparu que le mécontentement, suite à une vexation personnelle, ou pour signifier un désaccord plus général avec les décisions d'un chef, pouvait passer par une négligence plus ou moins longue des devoirs cérémoniels : soit de ce qui, précisément, exprime et reproduit au quotidien le statut du chef. Les chefs, inversement peuvent omettre de solliciter les contributions d'une par-

26. Montrer des qualités de chef (vakaturaga) impliquerait notamment d'être digne mais sans arrogance, avenant sans surcroît de familiarité, respectueux des manifestations de déférence et d'humilité des membres du vanua, peu enclin à la confrontation et à la colère, etc. Les habitants déploraient volontiers ce qu'ils appréhendaient comme les effets négatifs des changements culturels chez les jeunes chefs : "You see the sons and daughters of chiefs mingling with ordinary people. It's modernization so to speak. You see them being drunk in nightclubs, behaving very badly, getting punched, or punching somebody else. Ok? So that's a very big change. When you are a chief, or a son of a chief, or a daughter of a chief, you are supposed to act in a chiefly manner all the time!" (septembre 2009).

27. D'après J. Nation: "The 'ruled', whether it be within a household, a village or a vanua, have the unofficial prerogative of grumbling (kudrukudru) or gossiping (kakase)" (1978:21). 
tie du vanua, ou bien redistribuer certaines attributions, s'ils estiment qu'un individu ou un clan est plus capable ou plus méritant. Une contestation peut aussi naitre, non pas autour de l'action d'un chef, mais parce que des membres de son mataqali ou tokatoka estiment être les vrais héritiers du titre. Ce type de querelle fréquente peut avoir des degrés de gravité et des expressions variables, et peut être éventuellement surmonté par le biais des cérémonies du pardon (i soro).

Les motifs d'insatisfaction ou d'inquiétude n'empêchent pas le sentiment de respect (vakarokoroko) pour les chefs de Rewa, et en particulier pour les détenteurs de titres prestigieux (que même les personnes de Rewa vivant en ville et éloignées des problématiques du vanua estiment devoir servir, veiqaravi), et la fierté d'appartenir à une province où le protocole entourant les chefs est réputé exigeant et bien préservé. Alors que la province de Tailevu, par exemple, se révèlerait plus transformée et occidentalisée, de l'avis d'habitants de Rewa. À Rewa, la hiérarchie est régulièrement présentée comme une caractéristique culturelle " authentiquement fidjienne ", équilibrée par la notion de respect mutuel (veidokai) et le concours de tous à l'harmonie sociale, et non comme une aberration passéiste, servant les intérêts de quelques familles ayant profité de l'administration coloniale pour essentialiser leur domination. Dans le cas du Roko Tui Dreketi, chef du vanua de Rewa, mais aussi du vaste matanitu de Burebasaga, des villageois ne cachent pas leur satisfaction de servir un chef si important, quand d'autres vanua sont privés d'un chef suprême, et qui plus est quand la Dame (marama) montre de très prestigieuses connections familiales avec Bau. Par ailleurs, en plus du respect témoigné aux titres, la personnalité et les réalisations des chefs de Rewa suscitent fréquemment des commentaires positifs. Par exemple, les personnes interrogées louent volontiers les efforts du Roko Tui Dreketi pour entretenir les liens ("keeping the connections alive ") avec toutes les parties du vanua, notamment par des visites des villages, ou par des apparitions et contributions aux fềtes familiales des chefs de tikina. C'est aussi pour représenter toutes les parties du vanua que Ro Teimumu Kepa se rendrait à la fois aux services religieux catholiques et méthodistes à Lomanikoro (cf. Fiji Times Online, 27/12/09, sur la bénédiction de la nouvelle résidence du Roko Tui Dreketi par un prêtre catholique et un pasteur méthodiste). Les personnes de Rewa, d'autre part, se déclarent souvent fières d'avoir pour chefs principaux une femme et un homme instruits, que leurs compétences rendent spécia- lement aptes à assumer le rôle de guides pour la population du vanua. L'attitude cordiale et sans artifices du Roko Tui Dreketi et du Vunivalu, conséquence, selon les informateurs, de contacts prolongés avec la vie urbaine et les idées modernistes qui y circulent, suscite aussi une certaine admiration, même s'il est impératif (pour le rayonnement des titres) d'en limiter l'expression. Des informateurs estiment même que c'est justement parce que certains chefs sont sûrs de leur statut, de la légitimité de leur pouvoir, de la solidité de leurs connections familiales et de leurs alliances, qu'ils peuvent se montrer plus flexibles avec le protocole : même s'il " cède la première coupe de yaqona" (celle qui est théoriquement réservée au rang le plus élevé) pour honorer un invité, un grand chef reste un grand chef, et tous les participants du rite le savent pertinemment.

En résumé, il est apparu dans les parties précédentes que le rôle des chefs locaux était l'objet d'interprétations variées, conflictuelles, évolutives. D'une façon générale, les chefs doivent composer avec la double nécessité de protéger leur statut et de ne pas faire montre d'un dédain décalé avec l'époque, de s'inscrire dans la tradition et d'exposer les signes d'une ouverture au monde, au changement, au progrès technologique ${ }^{28}$, et réaliser l'équilibre entre une inscription dans le vanua et des activités extérieures source de prestige. Un autre point délicat implique l'articulation des devoirs traditionnels et des responsabilités nationales, auxquelles leur statut social semble les destiner, et qui sont aussi une manière de compenser la faiblesse d'une situation locale, voire de préparer le terrain pour l'obtention d'un titre : quoi de mieux, en effet, qu'une élection au Parlement pour prouver la popularité d'un prétendant au titre de chef ? Le Roko Tui Dreketi se trouve pour sa part soumis aux nécessités contradictoires de préserver son statut par une forme d'immobilité cérémonielle ("She should be sitting, beautify herself, not working, going to the ground ") et de séparation d'avec la société, et d'agir conformément à son rang, en contribuant largement aux cérémonies, en redistribuant des richesses, en apportant des améliorations concrètes dans la vie du vanua : toutes choses qui peuvent s'acquérir par le biais de la politique et du travail en général, comme nous l'avons vu plus haut. Même si la définition de la " juste manière d'être chef " divise la population de Rewa, les enquêtes suggèrent in fine une forme d'attachement à la noblesse locale, dont l'unité, même considérée comme structurellement fragile, conditionne celle du vanua dans son ensemble. Les villageois ont aussi affirmé respecter les chefs des autres provinces et

28. Un homme ayant participé à la campagne électorale de Ro Teimumu Kepa en 2006 fit le commentaire suivant : «It was 2006, we had modern technology in our campaign, our campaign team. We had projectors, we had stereos [...] she won the heart of the people not only by using her Roko Tui Dreketi status... forget that! She won the heart by... Ro Teimumu, technology wise chief? " (février 2009). 
le grand conseil des chefs, mais une très forte différence est faite entre les chefs locaux et ces chefs étrangers, que la population n'a pas le devoir hérité de servir. Tout en insistant sur l'importance du système politique coutumier, notamment en tant qu'héritage culturel et facteur d'unification pour les Fidjiens, les habitants évoquent aussi sa "dégradation " ou son "affaiblissement " et de pressants besoins de réforme.

\section{Les chefs perdent-ils leur mana?}

Tous les habitants interrogés sur ce thème soulignent que le système politique coutumier a été affaibli ces dernières années par le fait que de nombreux titres, et en particulier plusieurs des titres principaux (notamment les titres suprêmes de Bau et des îles Lau), soient désormais vacants (Fiji Times, 17/10/2006 ; Fiji Times Online, 20/04/2007). Un enseignant retraité décrivait la situation en ces termes :

"That is one of the difficulties that we are facing now throughout Fiji. [...] The majority of the titles, chiefly titles, in Fiji are still vacant. And that in a way weakens the system. The chiefly system. Because if you are not installed formally, then your voice will not been heard in the Fijian syrup. You don't have that kind of mana, so to speak. When you are installed formally, in the traditional manner, and you hold the title, then your voice will be heard. People will respect you. Unfortunately the majority of Fijian titles, right from the paramount chief, right down to the lowest rank, the mataqali. Probably I can say about $60 \%$, even $70 \%$ are still vacant. " (Nakasi, septembre 2009)

Pour certains informateurs, cette situation s'expliquerait d'abord par l'augmentation de la valeur pécuniaire des terres, particulièrement dans la partie Ouest du pays, où se concentre une grande partie de l'infrastructure touristique et où la location de parcelles génère le plus de profits. Parce que les chefs reçoivent une part substantielle des revenus fonciers de leur mataqali ou yavusa ${ }^{29}$, la compétition pour les titres se serait considérablement accrue, aboutissant à des impasses, et à l'impossibilité de parvenir à des accords dans de nombreux cas (cf. Fiji Sun, 29/07/1986). Dans d'autres régions (comme dans la province de Rewa) où la valeur des terres est moindre, les conflits relèveraient davantage d'une compétition statutaire classique, qui se réglait autrefois par la guerre, avec la mise en avant de principes de légitimité variables par les différents prétendants, mataqali, ou tokatoka. Des habitants évoquent dans ce cadre les ambitions démultipliées de certains chefs, que leurs succès politiques ou professionnels pousseraient à revendiquer des titres même avec une position familiale faible (en étant issus d'un second mariage du précédent détenteur du titre, ou d'une branche cadette), et leur capacité à trouver aussi, grâce à ces succès, des soutiens élargis au sein du vanua. Lors des entretiens, cette situation de vacance du pouvoir fut toujours présentée comme négative, les conséquences étant entre autres : une profonde division à l'intérieur des vanua concernés (dans les familles de chefs et dans la population), un affaiblissement du pouvoir et de la légitimité du grand conseil des chefs, où des vanua aussi fondamentaux que Bau et les Lau ne peuvent " parler d'une seule voix ", une perte de respect pour les chefs en général. Selon certains villageois, l'assouplissement des tabous hiérarchiques et la multiplication des unions entre personnes nobles et non nobles auraient également contribué à affaiblir la position des chefs :

"It is also the marriages that so create... Fifty years ago, blue blood marry a blue blood. Ok? Now, that has changed. So we can have a man of a chiefly household marry only a ordinary woman from... a commoner, so to speak. That was not done, years ago. [...] That is diluting the chiefly system in a way. That mana that was seen before [...] is no longer there, because... ob! he's just a commoner from that side. " (Septembre 2009)

Pour d'autres, des chefs auraient profité des avantages de leur position coutumière, notamment fonciers, en oubliant leurs devoirs de réciprocité, suscitant par là même la méfiance de la Terre et des comportements subversifs et désinvoltes (cf. Keith-Reid, 1982 : 14 ; Fiji Times, 9/04/2007 ; Fiji Sun, 16/04/2007). D'une façon générale, les révélations des médias sur le train de vie de certains chefs ou sur des opérations financières peu claires (Lawson, 1991: 245-248), et la valorisation croissante du principe de performance par rapport au statut hérité, semblent avoir fragilisé la position des chefs en tant que classe sociale, et accru la vigilance populaire sur leurs activités. En outre, les progrès de l'instruction publique amènent un nombre grandissant de Fidjiens diplômés à briguer de hautes responsabilités et à dénoncer, même discrètement, l'inaptitude de quelques chefs promus dans la haute administration ou à des postes politiques en raison de leur seule origine familiale :

"I'm proud to say that Fiji has really advanced in the education field. Compared to other Pacific islands. So maybe fifty years ago, it would have been different. They could see that their chief is not performing his job properly, probably they just follow what... But not now, it's different." (Ibid.)

Il n'est pas impossible non plus que le discours politique de feu Sakeasi Butadroka (fonda- 
teur du Fijian Nationalist Party dans les années 1970 et originaire de Rewa), pour qui les chefs avaient trahi leur peuple en cédant Fidji aux Britanniques, puis en acceptant une indépendance conduisant au partage du pays avec les Indo-Fidjiens, ait alimenté une certaine défiance populaire vis-à-vis des chefs, dont on aurait toujours les traces (Rutz, 1995 : 82-83).

Le fait que des chefs soient ou aient été engagés dans des partis politiques est aussi régulièrement présenté comme une cause d'affaiblissement du système politique coutumier. Comme on l'a vu, la politique nationale, fondée sur des codes de conduite et des rapports hiérarchiques entièrement différents de ceux du vanua, amènerait nécessairement des commentaires, des situations, qui ternissent l'image des chefs et heurtent leurs sujets. Des habitants expliquent d'autre part que les divisions de plus en plus visibles entre les membres du grand conseil des chefs (liées en particulier à des rivalités internes entre familles et matanitu), et l'ouverture à des personnalités non nobles, comme Sitiveni Rabuka ${ }^{30}$, ont fait perdre du crédit à l'institution ( $c f$. Fiji Sun, 14/04/2007 c). Les analyses dénonçant l'esprit communautariste des chefs et le fait que certains aient pu fait obstacle à la construction de l'unité nationale (Fiji Times, 1/09/2004) ne sont, par contre, jamais apparues dans les entretiens réalisés à Rewa. Sur le terrain, la nation multiethnique fut plus souvent présentée comme une fatalité que comme un projet désirable, et est apparue associée au spectre d'un délitement de l'identité et des traditions fidjiennes. Au cours des entretiens, des habitants, issus de milieux socioprofessionnels et de clans divers, ont expliqué que le système politique coutumier devait évoluer en profondeur pour ne pas risquer de disparaittre, deux hommes, l'un enseignant et l'autre militaire, le considérant même déjà comme moribond. Selon une idée communément admise, les chefs devraient avoir une conscience claire de leurs responsabilités et montrer " de vraies qualités de leaders " pour garder la confiance et le respect des Fidjiens; surtout à une époque où l'extension des villes, la mondialisation culturelle, les mutations économiques, représentent autant de dangers pour la préservation des coutumes. De fait, certains habitants de Rewa ont estimé que l'affaiblissement général du système des chefferies n'était en rien l'effet exclusif des erreurs commises par les chefs. Il serait aussi imputable à des changements idéologiques (accent mis sur la performance et le mérite individuel plutôt que sur les statuts et les qualités hérités), au fait que les Fidjiens tendent à négliger leurs devoirs traditionnels (qu'ils associent plus facilement leur identité personnelle à des catégories socioprofessionnelles, à des groupements reli- gieux, qu'à une position à l'intérieur du vanua), et au fait que le capitalisme rebatte les cartes, en permettant d'acquérir pouvoir et entregent sans un rang élevé. Les principaux enjeux, pour les chefs, deviendraient désormais : comment incarner au mieux les nouvelles aspirations, les nouvelles règles du jeu de la société fidjienne ? Comment être modernes sans se renier, sans abîmer l'aura de titres qu'une partie des Fidjiens continue de tenir pour précieux ? Comment rester influents dans un monde qui change, et ne pas voir les marques de respect, les rituels de la chefferie, changés en folklore politiquement inefficace?

Tout en pointant les erreurs commises ou le besoin de réformes, les habitants de Rewa ont aussi fait part du respect dans lequel ils tenaient les chefs en général et le grand conseil des chefs, même si le sentiment d'allégeance s'applique par contre exclusivement aux chefs locaux. En d'autres termes, reconnaitre et honorer le statut d'un dignitaire étranger, notamment s'il est apparenté aux chefs de Rewa, n'impliquerait nullement un devoir d'obéissance : seuls les chefs de Rewa peuvent ordonner aux gens de Rewa. Pour une large partie des villageois, la chefferie représente d'abord un caractère culturel distinctif à protéger, allant de pair avec une organisation sociale communautaire fondée sur le partage, opposée à l'individualisme et à la course au profit des pays occidentaux. Par ailleurs, des habitants insistent sur le fait que le système des chefferies contribue à donner un rôle social à chacun et à unir les différents clans autour de références communes et de buts communs. Le système politique coutumier fait que les Fidjiens sont des guerriers, des prêtres, des mataki (émissaires, correspondants locaux), pour des clans de leur vanua ou pour des clans étrangers, et qu'il est nécessaire de se rassembler pour collecter ou redistribuer des biens cérémoniels, pour honorer un dignitaire, pour choisir le prochain détenteur du titre. Les liens de parenté entre les chefs sont aussi vus comme des vecteurs de communication et de paix, comme un rempart contre les conflits qui peuvent facilement enflammer et diviser les Fidjiens ("blood is thicker than water"). Des chefs au sens large, un habitant, issu d'une famille de haut rang de Lomanikoro, déclarait :

\footnotetext{
"Chiefs are there for the people as symbol of unity, togetherness, prosperity"
}

et aussi :

"they are the centre of unity for the Fijian people. " (Décembre 2007)

Selon certains informateurs, l'affaiblissement du pouvoir des chefs et de la coutume serait di- 
rectement responsable d'une dégradation des relations au sein de la communauté mélanésienne, notamment après le coup d'État de 2006, tensions que la pratique de la religion chrétienne permettrait d'endiguer, sinon de surmonter efficacement. En outre, des habitants appartenant à des clans de statut noble et non noble ont fait part de leur crainte de voir advenir, dans le sillage du coup d'État de 2006, une sorte de nivellement du statut des chefs et leur remplacement par des agents du gouvernement :

"Chiefs, they will be classed all the same. [...] Only chief here is... what... the D.O. [District Officer] and the P.A. [Provincial Administrator]! » (Lomanikoro, septembre 2009)

Une majorité d'habitants a d'autre part exprimé son " respect " pour le grand conseil des chefs, aujourd'hui suspendu, en insistant sur son importance pour défendre les intérêts de la communauté mélanésienne, notamment sur la délicate question des terres. Des informateurs ont avancé l'idée que le grand conseil des chefs était la seule instance capable d'unir les Fidjiens en temps de crise : que les Fidjiens, par respect et par allégeance pour leurs dignitaires, pouvaient surmonter leurs divisions si le conseil "parlait d'une seule voix ». Des villageois ont aussi laissé entendre que le mana du grand conseil des chefs était nécessaire au bon fonctionnement du pays, notamment pour guider et légitimer les décisions du gouvernement. On constate toutefois que la nature exacte des attributions du grand conseil des chefs reste floue pour une large partie des habitants, et surtout que la population se réfere avant tout aux positions et décisions de ses propres chefs, plutôt qu'au conseil lui-même.

Résumons maintenant ce qui a été exposé dans la deuxième partie. Au bout du compte, les personnes consultées à Rewa n'ont qu'exceptionnellement mis en cause l'utilité et la légitimité de la présence de chefs dans la société fidjienne, et des mécanismes sociaux (prestations des clans, étiquette, privilèges) qui expriment leur statut et organisent leur séparation d'avec les " gens du commun ". Même si les enquêtes ne peuvent donner qu'une idée générale des opinions existantes, une part importante de la population paraît considérer que les chefs jouent un rôle clef pour la construction de l'unité et de la prospérité du vanua, en association étroite avec le pouvoir religieux chrétien. Comme on l'a vu, les inquiétudes ou critiques formulées au sujet des chefs de Rewa évoquent souvent plus un attachement à leurs personnes et à leurs titres qu'une rébellion tendant vers le désir d'une "société sans chefs " (Rabuka, 2007), même si l'exigence de réciprocité et la dénonciation de fautes ou de manques sont aussi présentes. Si les différences statutaires et l'autorité locale des chefs ne semblent pas réellement remises en cause, l'approche de leurs rôles nationaux divise clairement la population. La question du rôle national des chefs locaux est de loin la plus passionnément débattue, à la mesure des enjeux qu'elle soulève, des pouvoirs que peut offrir l'inclusion à l'appareil d'État. Il est apparu au fil de l'article que le Roko Tui Dreketi constituait à différents titres un chef à part, et que l'importance cruciale de ses fonctions cérémonielles rendait une activité politique spécialement difficile à admettre. La mise en danger de son mana dans le chaos égalitaire de la " politique à l'anglaise " fragilise tout l'équilibre de la chefferie, vue comme une construction constamment menacée par des forces centrifuges, par les ambitions internes et externes, par la déliquescence de la coutume, et que les rituels et l'Église contribuent à perpétuer. Que des chefs de moindre importance se risquent en politique comporte, à l'évidence, moins de risques locaux. Il est clair cependant que les approches traditionalistes, voulant " ramener les chefs dans les villages » au nom de la coutume, font aussi intervenir des stratégies personnelles, une compétition statutaire s'exerçant sous le vernis des rituels hiérarchiques. Ces discours tendent au fond à "dégager la place ", ou bien aux membres non titrés des familles nobles, ou bien à des gens de la Terre qui font reposer leur influence sur leurs qualités personnelles, leur éducation, leur pouvoir économique et leurs réseaux. Pour les détenteurs de titres a contrario (et pour une partie des personnes qui leur font allégeance), une fonction politique ou bureaucratique peut être le moyen de se préserver une vraie capacité d'influence et d'être en mesure de tenir leur rang, face aux chefs rivaux et aux nouvelles élites non nobles : de continuer à redistribuer, à nourrir, à donner plus que les autres. Par ailleurs, et même si un seul villageois a clairement mentionné ce point, on peut soupçonner que la qualité de femme, qui plus est de confession catholique, de Ro Teimumu Kepa, a pu représenter une difficulté additionnelle pour faire admettre ses propositions de changement du rôle du chef suprême, et la concentration en ses mains de pouvoirs à la fois locaux et nationaux.

\section{Conclusion}

Cet article a examiné comment les questionnements actuels sur le rôle des chefs fidjiens peuvent s'exprimer, ou bien être perçus, à l'intérieur d'un vanua (ainsi que les enjeux qui sous-tendent la critique du pouvoir traditionnel quand elle existe), et a aussi mis en valeur certains des facteurs contribuant à transformer la position des chefs. Nous avons entrevu par exemple que le développement économique, les progrès d'une 
idéologie de la performance ou un meilleur accès à l'éducation favorisaient la mobilité statutaire des gens de la Terre et des cadets des chefferies, ainsi qu'une forme de contrôle populaire sur l'action des chefs. L'histoire s'est toutefois accélérée après décembre 2006 : en suspendant les activités du grand conseil des chefs, le gouvernement issu du coup d'État a manifesté sa volonté d'organiser la dissociation de deux sphères politiques, coutumière et nationale, qui depuis la période coloniale (et même à cause d'elle) avaient été largement imbriquées. Aujourd'hui, les chefs qui exercent des responsabilités politiques ont été incorporés en tant quiindividus à l'administration d'État, et non en tant que représentants d'un vanua ou d'une famille donnés. Les célébrations nationales accordent aussi une moindre place à l'étiquette et aux rituels mélanésiens, tout en reflétant l'extension spectaculaire du rôle de l'armée. En l'absence du grand conseil des chefs, le gouvernement et les autorités militaires se retrouvent seuls responsables de la définition officielle de l'identité et des droits de la communauté autochtone, et seuls « faiseurs de chef " pour le choix du Président, comme l'a montré la nomination de Ratu Epeli Nailatikau en novembre 2009.

Des commentateurs ont suggéré que les réformes engagées par les militaires pouvaient marquer le début d'une remise en cause plus générale du pouvoir des chefs, cette fois au sein des villages, et par leurs propres sujets coutumiers. Il n'est pas exclu que les atteintes symboliques au statut des chefs (remarques volontairement irrespectueuses des dirigeants de l'armée aux chefs opposants, interrogatoires, manifestations de puissance au sein des chefferies), et les possibilités plus restreintes de défendre les intérêts du vanua, en l'absence du grand conseil des chefs, puissent avoir à terme des conséquences sur les relations chefs-sujets. La longévité du gouvernement, en dépit des provocations, des "blasphèmes ", est susceptible d'être interprétée comme un signe d'effondrement du mana des chefs, et d'inciter à d'autres subversions, locales cette fois, des tabous hiérarchiques. L'enquête conduite à Rewa montre néanmoins que les chefs sont encore dans certaines régions considérés comme des agents cruciaux de l'équilibre et du progrès de la société, tout en étant tenus à une forme de réciprocité et d'efficacité. Encore une fois, nous avons vu à Rewa que les reproches adressés aux chefs peuvent parfois désigner en creux l'importance fondamentale de leur présence et de leur mana pour les communautés locales, et aussi l'importance accordée à la " coutume fidjienne ", que beaucoup estiment menacée par la mondialisation culturelle, l'exode vers les villes, la vacance des titres. Nous avons vu d'autre part que les chefs sont amenés à s'adapter aux mutations sociales et politiques en cours, et à construire dans cette optique un équilibre délicat, et constamment négocié avec leurs propres sujets, entre empathie et distinction.

En définitive, les changements sociopolitiques actuels, qu'ils relèvent d'une évolution lente, ou des réformes apportées par le régime en place, posent des défis aux chefs autant qu'ils ouvrent des possibilités. Et notamment celle de remettre en cause des équilibres hiérarchiques entre titres ou provinces certainement moins stabilisés, ou admis, que les rituels du grand conseil des chefs pouvaient le laisser croire ${ }^{31}$. Il restera à voir si ces réagencements parviennent à s'imposer comme une "nouvelle tradition", et aussi si un projet national où la référence aux chefs et à leur mana est absente ou secondaire réussit, sur le long terme, à rassembler les Mélanésiens.

\section{Remerciements}

Je tiens à exprimer tous mes remerciements à la Marama Bale Na Roko Tui Dreketi, au Turaga Vunivalu et aux habitants de Rewa qui mont accueillie et épaulée pour les enquêtes de terrain entre 2007 et 2011. Je remercie aussi la Fondation Singer Polignac, les membres du programme collectif LocNatPol soutenu par l'ANR, le AXA Research Fund, ainsi que les chercheurs qui ont bien voulu relire cet article. Merci en particulier à Simonne Pauwels, du CREDO, pour ses commentaires sur le présent article. Je reste bien sûr seule responsable des interprétations tirées des matériaux d'enquête.

\section{BIBLIOGRAPHIE}

Bose Apolosi et Fraenkel Jon, 2007. Whatever happened to Western separatism?, in S. Firth and J. Fraenkel (eds), From election to coup in Fiji, the 2006 campaign and its aftermath, Canberra, Australia National University E Press/Asia Pacific Press, Suva, IPS Publications, pp. 225-242.

BRISON Karen, 2001. Constructing identity through ceremonial language in rural Fiji, Ethnology 40, 4, pp. 309-327.

\footnotetext{
31. De nettes fissurations sont apparues à partir de fin 2008 dans le matanitu Burebasaga, vaste construction politique datant de la période coloniale. Des chefs et des villages entiers se sont détournés du Roko Tui Dreketi et ont exprimé cérémoniellement leur allégeance à Franck Bainimarama (sur Namosi et Kadavu : Fiji Sun Online, 31/10/2009 et 26/01/2010). Le Vunivalu de Rewa lui-même choisit de manifester publiquement son soutien au gouvernement en se désolidarisant du chef suprême, un véritable défi au protocole, qui fut interprété à Rewa comme la poursuite d'une rivalité statutaire d'origine précoloniale (Fijilive, 15/12/2008).
} 
Derrick Ronald Albert, 2001 ( $1^{\text {st }}$ ed. 1946). $A$ history of Fiji, Suva, The Government Press.

Drova TAdrau Nemani, 1985. Oral traditions and identity: a case study from Rewa, Fiji, in D. Routlege (ed.), Papers in Pacific History, Suva, The University of the South Pacific, SSED Student Papers Series 1, pp. 56-73.

Fiji Government Online Portal, 2007 (I $2 / 04$ ). Address by PM Bainimarama after the Great Council of Chiefs Meeting (consulté sur internet le 21/02/2009, http://www. fiji.gov.f/).

Fiji Islands Bureau of Statistics, 2010. Key statistics : June 2010. Population of Fiji by ethnicity. 2007 Census of Population and Housing (consulté sur internet le 9/05/2011, http://www.statsfiji.gov.ff/Census2007/census07_index2.htm).

FijILIVE, 2008 (I $5 /$ I 2 ). Vunivalu of Rewa turns up at barracks (http://www.fijilive.com/news_ new/index.php/news/show_news/11683).

FIJI SUN, 1986 (29/07). This chief is for the people.

—, 2007 (14/04 a). GCC and Fiji's woes.

—, 2007 (14/04 b). Chiefs are the cause.

-, 2007 (14/04 c). Still a role for the chiefs.

—, 2007 (16/04). Province backs PM's decision.

FIJI SUN ONLINE, 2009 (3I/Io). Ratu Suliano breaks silence (http://www.fijisun.com.fj/ main_page/view.asp?id=28827).

—, $2010(26 / 01)$. Kadavu defies paramount chief (http://www.fijisun.com.fj/main_page/ view.asp?id=33287).

FIJI TIMES, 2004 (or/o9). Chiefs losing their mana.

—, 2006 (17/10). Title-holders must register now.

-, 2007 (09/04). Chiefs' complex.

-, 2007 (14/04). Pillar of strength.

-, 2009 (1/04). Chiefly system a threat.

FIJI TIMES ONLINE, 2007 (20/04). Most chiefs breach tradition (http://www.fijitimes.com/ story.aspx? $\mathrm{ref}=$ archive\&id=61053).

—, 2008 (24/03). Rabuka backs GCC changes (http://www.fijitimes.com/story. aspx?id=84667).

—, 2009 (27/12). Chiefly home in Rewa blessed (http://www.fijitimes.com/story. aspx?id=136196).

Firth Stewart and Fraenkel Jon, 2007. The cycles of party politics, in S. Firth et J. Fraen- kel (eds), From election to coup in Fiji, the 2006 campaign and its aftermath, Canberra, Australia National University E Press/Asia Pacific Press, Suva, IPs Publications, pp. 64-77.

Fraenkel Jon, 2007. Addendum. The Fiji coup of December 2006. Who, what, where and why?, in S. Firth and J. Fraenkel (eds), From election to coup in Fiji, the 2006 campaign and its aftermath, Canberra, Australia National University E Press/Asia Pacific Press, Suva, IPS Publications, pp. 420-449.

Hocart Arthur Maurice, ms. The heart of Fiji, Turnbull Library, Wellington, NouvelleZélande.

Keith-Reid Robert, 1982 (décembre). The future of the chiefs, Islands Business, pp. 14-20.

Lawson Stephanie, 1991. The failure of democratic politics in Fiji, Oxford, Oxford University Press.

Macnaught Timothy J., 1974. Chiefly civil servants? Ambiguity in district administration and the preservation of a Fijian way of life, 1896-1940, Journal of Pacific History 9, 1, pp. 3-20.

Madraiwiwi Joni (Ratu), 2006. Keynote address. Governance in Fiji: the interplay between indigenous tradition, culture and politics, in S. Firth (ed.), Globalisation and governance in the Pacific Islands. State, Society and Governance in Melanesia, Studies in State and Society in the Pacific 1, The Australian National University E Press, pp. 289-296.

Nation John, 1978. Customs of respect: the traditional basis of Fijian communal politics, Canberra, Australian National University, Development Studies Centre, Monograph 14.

NayaCaKalou Rusiate, 1985. Leadership in Fiji, Suva, University of the South Pacific, Oxford University Press (1 ${ }^{\text {st }} 1975$. John Sands, Halstead Press Division, Australie).

Newbury Colin, 2006. Bose Vakaturaga: Fijis Great Council of Chiefs, 1875-2000, Pacific Studies 29, 1/2, pp. 82-127.

Newland Lynda, 2009. Religion and politics: the Christian churches and the 2006 coup in Fiji, in S. Firth, J. Fraenkel, B. V. Lal (eds), The 2006 military takeover in Fiji. A coup to end all coups?, Canberra, Australia National University E Press, pp. 187-207.

Norton Robert, 2009. The changing role of the Great Council of Chiefs, in S. Firth, J. Fraenkel, B. V. Lal (eds), The 2006 military takeover in Fiji. A coup to end all coups?, Canberra, Australia National University E Press, pp. 97-115. 
Qetaki Alipate, 2011 (22/01). Public notice. New rent distribution policy. Native Land Trust Board, Press release (consulté en ligne le 9 mai 2011, http://www.nltb.com.f/news_ press_releases.html).

Rabuka Sitiveni, 2007 (15/04). A chiefless Fijian society, Fiji Times Online (http://www.fijitimes. $\mathrm{com} /$ story.aspx? ref=archive\&id=60781).

RaJ Rishi, 2004. Fiji Islands: Flood Management, Rewa River Basin, World Meteorological Organization and Global Water Partnership, Associated Programme on Flood Management, edited by the Technical Support Unit.

Rutz Henry J., 1995. Occupying the headwaters of tradition: rhetorical strategies of nation making in Fiji, in R. J. Foster (ed.), Nation Making: Emergent Identities in Postcolonial Melanesia, Ann Arbor, University of Michigan Press, pp. 71-93.

SaHlins Marshall, 1985. Islands of History, Chicago, The University of Chicago Press.

—, 1991. The return of the event, again. With reflections on the beginnings of the great $\mathrm{Fi}$ jian war of 1843 to 1855 between the kingdoms of Bau and Rewa, in A. Biersack (ed.), Clio in Oceania. Toward a Historical Anthropology, Washington, Smithsonian Institution Press, pp. 37-99.

—, 2004. Apologies to Thucydides, Chicago, The University of Chicago Press.

Saumaki Baro, 2007. Bose ni vanua and democratic politics in Rewa, in S. Firth et J. Fraenkel (eds), From election to coup in Fiji, the
2006 campaign and its aftermath, Canberra, Australia National University E Press/Asia Pacific Press, Suva, IPS Publications, pp. 213224.

SCARr Deryck, 1970. A Roko Tui for Lomaiviti: the question of legitimacy in the Fijian Administration, 1874-1900, Journal of Pacific History 5, 1, pp. 3-31.

Tomuinson Matt, 2002. Sacred soil in Kadavu, Oceania 72, 4, pp. 237-257.

Tudrau-Tamani Ana, 2006 (September). Ro Teimumu. Born a leader, Marama, pp. 14-18.

WARD Gerard and KingDon Elizabeth (eds), 1995. Land, custom and practice in the South Pacific, Cambridge, Cambridge University Press.

Watter Michael A., 1978. Analysis of Fijian traditional social organization: the confusion of local and descent grouping, Ethnology 17, 3, pp. 351-366.

YabaKi Akuila (révérend), 2006 (16/11). Chiefs must stand against political manipulation, Fiji Times, pp. 10-11.

\section{Archives}

ANF : Archives nationales de Fiji, fonds du Colonial Secretary Office (cso), Carnavon street 25, Suva, Fidji.

APM : Archives des pères maristes, Via Alessandro Poerio 63, Rome, Italie. 